\title{
Instability of the salinity profile during the evaporation of saline groundwater
}

\author{
By ANDREJ T. IL'ICHEV'1, GEORGE G. TSYPKIN ${ }^{2}$, DAVID \\ PRITCHARD $^{3}$ AND CHRIS N. RICHARDSON ${ }^{4}$ \\ ${ }^{1}$ Steklov Mathematical Institute, Russian Academy of Sciences, Gubkina Str. 8, 119991 \\ Moscow, Russia. Email: ilichev@mi.ras.ru. \\ ${ }^{2}$ Institute for Problems in Mechanics, Russian Academy of Sciences, Av. Vernadskogo 101, \\ 119420 Moscow, Russia. Email: tsypkin@ipmnet.ru. \\ ${ }^{3}$ Department of Mathematics, University of Strathclyde, 26 Richmond Street, Glasgow G1 \\ 1XH, Scotland. Email: dtp@maths.strath.ac.uk. \\ ${ }^{4}$ BP Institute for Multiphase Flow, University of Cambridge, Madingley Rise, Cambridge \\ CB3 0EZ, UK. Email: chris@bpi.cam.ac.uk
}

(Received 18 June 2008)

We investigate salt transport during the evaporation and upflow of saline groundwater. We describe a model in which a sharp evaporation-precipitation front separates regions of soil saturated with an air-vapour mixture and with saline water. We then consider two idealised problems. We first investigate equilibrium configurations of the fresh-water system when the depth of the soil layer is finite, obtaining results for the location of the front and for the upflow of water induced by the evaporation. Motivated by these results, we develop a solution for a propagating front in a soil layer of infinite depth, and we investigate the gravitational stability of the salinity profile which develops below the front, obtaining marginal linear stability conditions in terms of a Rayleigh number and a dimensionless salt saturation parameter. Applying our findings to realistic parameter regimes, we predict that salt fingering is unlikely to occur in low-permeability soils, but is likely in high-permeability (sandy) soils under conditions of relatively low evaporative upflow.

\section{Introduction}

As human activity in many parts of the world puts an increasing demand on scarce water supplies, the quality of sub-surface water and the accumulation of contaminants in soil and in aquifers are of increasing social and environmental significance. An important set of problems concerns the accumulation of salt when groundwater is extracted for agricultural irrigation, or when civil engineering projects significantly affect the groundwater level. Such problems are prevalent in hot arid regions, including parts of Australia, India, southern Russia and Sahelian Africa, and climate change is likely to exacerbate them and to extend the affected areas (Schofield \& Kirkby 2003), with severe consequences for agriculture (Yeo 1999).

Evaporation from the surface draws water up through the soil: when this water evaporates, its load of salt is precipitated, and if the phase transition always occurs at the soil surface then a thick crust of salt may accumulate there. However, other scenarios are possible. For example, the evaporation front may not remain fixed at the surface, but instead descend through the soil. Additionally, the accumulation of salt near the surface means that the near-surface water becomes more saline and thus denser than that below 
it; this may lead to salt fingering and the redistribution of saline water deep into the ground (where it may, for example, contaminate the aquifer). Whether a gravitational instability occurs is important because it determines which of two distinct environmental problems must be addressed.

In this study, we aim to elucidate the basic physical mechanisms which control this process, rather than to incorporate all the various (and site-specific) physical, chemical and biological processes which occur in near-surface groundwater. To this end, we employ an approach based on conservation laws, Darcy's law for fluid motion, and equilibrium thermodynamical relations (Phillips 1991; Helmig 1997). This builds on a substantial body of research on salt transport in groundwater, though few previous studies have investigated travelling evaporation fronts. In particular, Wooding (1960), Wooding, Tyler \& White (1997), van Duijn et al. (2002) and Pieters \& van Duijn (2006) have used Darcian models to investigate instabilities driven by conditions fixed at the soil surface; Yakirevich, Berliner \& Sorek (1997) have modelled evaporation from the soil surface and the vertical profiles of soil saturation and ion content beneath it; Gowing, Konukcu \& Rose (2006) have used a quasi-steady model based on Richards' equation to predict vertical profiles including a descending evaporation front, and tested it against laboratory experiments. Most relevantly to the current study, Tsypkin \& Brevdo (1999) and Tsypkin (2003b) (see also Tsypkin 2003a) have developed one-dimensional models of Darcian flow with salt and heat transport and a moving evaporation front, but the stability of their solutions has not previously been considered.

This study is structured as follows. In $\S 2$ we discuss the evaporation of fresh groundwater, identifying the regimes in which system temperature and aquifer pressure control the position of the evaporation front, and obtaining estimates for upflow velocities. In $\S 3$ and $\S 4$ we investigate the more general situation when the groundwater is saline; we consider the linear stability of the salinity profile which develops beneath a front descending at a prescribed velocity, and use the results of the simpler problem considered in $\S 2$ to relate our findings to real-world salinisation problems. Finally, in $\S 5$ we summarise our findings and discuss some possible extensions to this work.

\section{Evaporation of fresh groundwater}

Groundwater evaporation and salt precipitation depend on the groundwater salinity, the velocity of groundwater upflow, the soil porosity and permeability, the system temperature and the air humidity. Estimates for the velocity of groundwater upflow can be obtained from a simplified model of the motion and evaporation of fresh water in a vertically bounded domain.

\subsection{Problem formulation}

We consider a physical system which consists of an aquifer or high-permeability watersaturated layer with constant pressure $P_{L}$, overlain by a comparatively low-permeability layer of soil with thickness $L$ (figure 1). We employ a Cartesian co-ordinate system with the origin located at the surface and the $z$-co-ordinate increasing downwards. A physical assumption which should be noted is that all components of the system are assumed to be at a constant system temperature $T_{0}$; we will discuss the validity of this assumption below.

We assume that the soil is an undeformable porous medium of constant porosity and that the evaporation process in the soil does not affect the state of the atmosphere, which is characterised by constant system temperature $T_{0}$, pressure $P_{a}$ and humidity $\nu_{a}$. (The humidity is defined as the ratio of the effective density of water vapour to that of air; 
Atmosphere:

humidity $\nu_{a}$, pressure $P_{a}$.

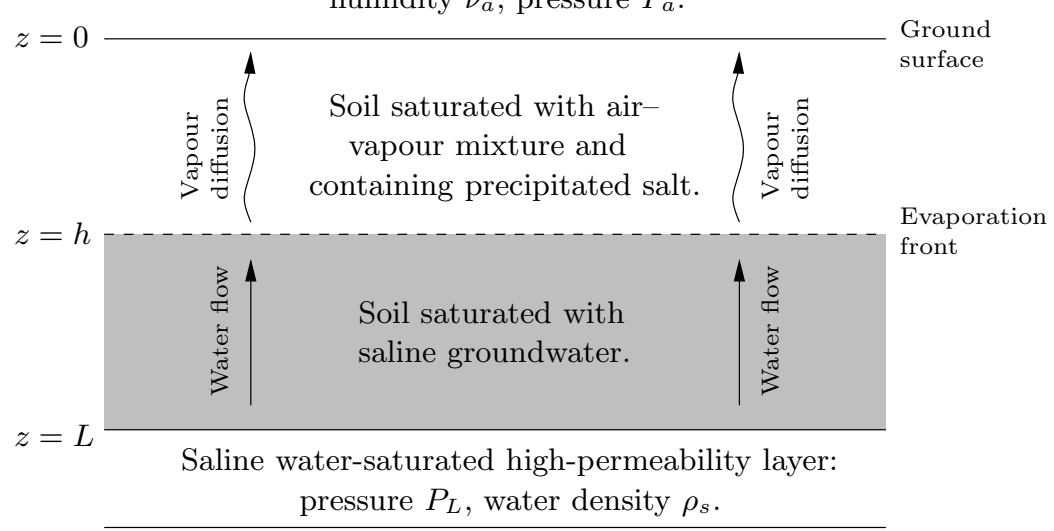

Figure 1. Schematic of the system considered; see text for definitions of the quantities. Note that in $\S 2$ the water is assumed to be fresh and there is no precipitated salt in the air-vapour region.

it is typically much less than unity.) The assumption of constant $\nu_{a}$ will break down in circumstances where the evaporative flux is high and a humid boundary layer forms above the soil surface, as can frequently be seen, for example, immediately after heavy rain on a hot day; we will comment below on the regimes in which this may occur.

For simplicity we follow other studies (Gowing et al. 2006; Pieters \& van Duijn 2006) by considering the soil to be isothermal, with the same constant system temperature $T_{0}$ as the ground surface. This assumption requires some discussion. Temperature variations in soils can be caused by the geothermal gradient, by seasonal warming or cooling, by the diurnal variation in atmospherical conditions, and also by the evaporation process: the magnitude of variation due to each of these factors can be estimated. A typical geothermal gradient is $0.03 \mathrm{~K} \mathrm{~m}^{-1}$, so over the vertical scales of a few metres considered here, this contribution may be neglected. The depth of penetration of the diurnal variation in ground surface temperature can be estimated from the heat diffusion equation as $L=$ $(T a)^{1 / 2}$, where $a$ is the thermal diffusion coefficient and where $T=12$ hours; typically $a \approx 0.3 \times 10^{-6}$ and so $L \approx 0.1 \mathrm{~m}$. It is therefore reasonable to neglect this contribution when the evaporation front has penetrated deeper than a few centimetres into the soil. The temperature change in the soil due to the seasonal warming and cooling of the atmosphere is a very slow process and takes a few months to cause changes of a few degrees in temperature if the depth is about $1 \mathrm{~m}$. Finally, variations in temperature due to the evaporation process are small compared to the geothermal energy flux, as has been shown by Tsypkin \& Brevdo (1999). Consequently, in the first approximation we can neglect the influence of temperature variation in the soil on the flow and transport processes considered here.

Evaporation occurs if the partial pressure of vapour in the air just above the soil surface is lower than the partial pressure of the saturated vapour. If this is the case then an air-vapour region is formed, and an evaporation front separates this zone from the zone saturated with water. Under these conditions, a diffusive flux of vapour takes place in the air-vapour zone, while water rises from the aquifer towards the evaporation front.

In the air-vapour domain the transport of water may be described using the diffusion 
equation that follows from mass conservation for vapour (Tsypkin and Brevdo 1999)

$$
\begin{gathered}
\frac{\partial \nu}{\partial t}-\nabla \cdot\left(D_{v} \nabla \nu\right)=0, \\
\text { where } \quad \nu=\frac{\rho_{v}}{\rho_{a}}=\frac{R_{a} P_{v}}{R_{v} P_{a}}, \quad P_{v}=A_{v} \rho_{v}, \quad P_{a}=A_{a} \rho_{a} .
\end{gathered}
$$

Here $T_{0}$ is the (absolute) system temperature; $A_{v, a}$ are constants related to the system temperature $T_{0}$ and the gas constants $R_{v, a}$ for vapour and air respectively through the relations $A_{v, a}=R_{v, a} T_{0} ; P_{a, v}$ are the partial pressures for air and water vapour respectively; $D_{v}$ is the vapour diffusivity, $\rho_{v}$ the mass of water vapour per unit volume of gas, and $\rho_{a}$ the density of air. Typical values (see e.g. Lide 2001) are $D_{v} \approx 2.4 \times 10^{-5} \mathrm{~m}^{2} \mathrm{~s}^{-1}$, $P_{a} \approx 10^{5} \mathrm{~Pa}, R_{a} \approx 287 \mathrm{~J} \mathrm{~kg}^{-1} \mathrm{~K}^{-1}, R_{v} \approx 461 \mathrm{~J} \mathrm{~kg}^{-1} \mathrm{~K}^{-1}$, and $T_{0} \approx 290$ to $330 \mathrm{~K}$.

In the domain saturated with water, we use mass conservation and Darcy's law, assuming that the groundwater density is constant, to write

$$
\begin{gathered}
\nabla \cdot \mathbf{v}=0 \\
\mathbf{v}=-\frac{k}{\phi \mu_{w}}\left(\nabla P-\rho_{w} g \mathbf{e}_{z}\right) .
\end{gathered}
$$

Combining equations (2.3) and (2.4) the equation for the pressure can be written as

$$
\nabla^{2} P=0 .
$$

In these equations $P$ is the pressure in the water, $\phi$ the porosity and $k$ the permeability of the soil, $\mathbf{v}$ the filtration velocity, $\rho_{w}$ the density of the groundwater, $\mu_{w}$ the dynamic viscosity of the groundwater and $g$ the acceleration due to gravity. Throughout, the subscripts $\mathrm{w}$, a and $\mathrm{v}$ will denote water, air and vapour respectively.

We assume that the evaporation occurs across a narrow front that separates the airvapour and water domains, and which we can treat as being of infinitesimal thickness. If we denote the pressure, partial vapour pressure and humidity at the front by $P_{*}, P_{v *}$ and $\nu_{*}$ respectively then the thermodynamic conditions at the evaporation boundary have the form

$$
\nu_{*}=\frac{R_{a} P_{v *}}{R_{v} P_{a}}
$$

where $P_{v *}$ can be related to the system temperature $T_{0}$ using the empirical correlation presented by Vukalovitch (1955),

$$
P_{v *}=10^{5} \exp \left[-7226.6\left(\frac{1}{T_{0}}-\frac{1}{373.16}\right)+8.2 \log \left(\frac{373.16}{T_{0}}\right)-0.0057\left(373.16-T_{0}\right)\right]
$$

where $T_{0}$ is measured in $\mathrm{K}$ and $P_{v *}$ in Pa. This was obtained by fitting to data over the range $273.16 \mathrm{~K} \leqslant T_{0} \leqslant 373.16 \mathrm{~K}$; other correlations could equally well be employed, such as that presented by Wagner \& Pruss (2002) which differs from (2.7) by less than $1 \%$ over the range of system temperatures considered here. It is useful to note some typical values: at $T_{0}=300 \mathrm{~K}, 320 \mathrm{~K}$ and $340 \mathrm{~K}$, we find $\nu_{*} \approx 0.022,0.065$ and 0.167 respectively.

The conservation of mass of water at the evaporation front can be expressed as

$$
\left(1-\frac{\rho_{v *}}{\rho_{w}}\right) \mathbf{V} \cdot \mathbf{n}=\mathbf{v} \cdot \mathbf{n}_{+}+D_{v} \frac{\rho_{a}}{\rho_{w}}(\nabla \nu) \cdot \mathbf{n}_{-}
$$

where $\mathbf{V}$ is the velocity of the evaporation front and $\mathbf{n}$ is a downward-pointing unit vector perpendicular to the front; the subscripts + and - indicate quantities which exist below or above the front, respectively. 


\subsection{Steady-state solution of the evaporation problem}

In order to illustrate the main properties of the evaporation process and to obtain estimates for the upflow velocity, we consider the one-dimensional steady vertical motion of water and vapour, as a function of the controlling parameters $P_{L}$ (the aquifer pressure) and $T_{0}$ (the system temperature).

As noted above, we assume for simplicity that the humidity is constant at the soil surface and unaffected by the vapour flux; furthermore, we assume that the (horizontal) water transport through the high-permeability aquifer is much larger than the (vertical) water flux from the aquifer into the soil. We also assume that a flux of groundwater from some distant source supports the temporally constant pressure in the aquifer. The boundary conditions then have the form

$$
P=P_{a} \quad \text { and } \quad \nu=\nu_{a} \quad \text { at } \quad z=0 ; \quad P=P_{L} \quad \text { at } \quad z=L .
$$

When $P_{L}>\rho_{w} g L$ we will refer to the aquifer as overpressured (so an upflow would be driven by the pressure even if the soil were completely saturated with water); when $P_{L}<\rho_{w} g L$ we will refer to the aquifer as underpressured.

This problem has a stationary solution in which the evaporation surface occurs at $z=h$ and the velocity $\mathbf{V}$ of the surface is zero. The location of the interface $h$ has to be found as part of the solution. The stationary one-dimensional solution to equations (2.1) and (2.5) can be written as

$$
\begin{gathered}
\nu=\nu_{a}+\frac{\nu_{*}-\nu_{a}}{h} z \quad \text { in } \quad 0<z<h ; \\
P=P_{a}+\frac{P_{L}-P_{a}}{L-h}(z-h) \quad \text { in } \quad h<z<L .
\end{gathered}
$$

Substituting solutions (2.10) and (2.11) into the boundary relation (2.8) at the evaporation front we obtain a quadratic equation for the unknown $h$,

$$
\frac{1-P_{a} / P_{L}}{1-h / L}-\frac{\rho_{w} g L}{P_{L}}-\frac{D_{v}}{\kappa} \frac{\rho_{a}}{\rho_{w}} \frac{\nu_{*}-\nu_{a}}{h / L}=0, \quad \text { where } \quad \kappa=\frac{k P_{L}}{\phi \mu_{w}},
$$

and thus, taking the unique positive solution for $h$

$\frac{h}{L}=-\frac{\lambda+\beta}{2}+\frac{1}{2} \sqrt{(\lambda+\beta)^{2}+4 \beta}, \quad$ where $\quad \lambda=\frac{P_{L}-P_{a}}{\rho_{w} g L}-1, \quad \beta=\frac{D_{v} \rho_{a}\left(\nu_{*}-\nu_{a}\right) P_{L}}{\kappa \rho_{w}^{2} g L}$.

The parameter $\lambda$ measures the deviation of the aquifer pressure from hydrostatic, while the parameter $\beta$ measures the importance of the upflow driven by evaporation, relative to a purely pressure-driven upflow.

We can use equation (2.13) to investigate how the location of the evaporation surface $z=h$ and the magnitude of the upflow velocity $v_{0}$ depend on the forcing conditions. In the steady state (when $\mathbf{V}=\mathbf{0}$ ) the upflow velocity, $v_{0}$, can be obtained from $(2.8)$ :

$$
v_{0}=\frac{\rho_{a} D_{v}\left(\nu_{*}-\nu_{a}\right)}{\rho_{w} h}
$$

Figure 2 illustrates the variation of $h$ and $v_{0}$ with system temperature $T_{0}$ and with the pressure $P_{L}$ in the aquifer, for a high-permeability system $\left(k=10^{-13} \mathrm{~m}^{2}\right)$ and a lowpermeability system $\left(k=10^{-18} \mathrm{~m}^{2}\right)$; recall that the system temperature $T_{0}$ controls $\nu_{*}$ through equations (2.6) and (2.7).

In the high-permeability system (figures 2 a and c), the dynamics are essentially pressure-controlled. When the aquifer is overpressured $\left(P_{L}>1.98 \times 10^{5} \mathrm{~Pa}\right), v_{0}$ varies 
(a)

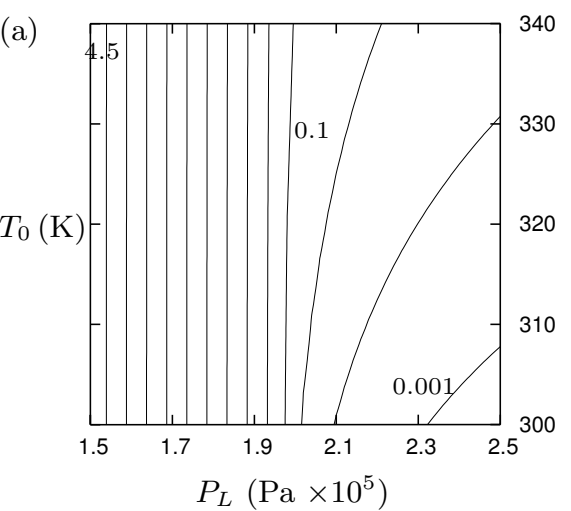

(c)

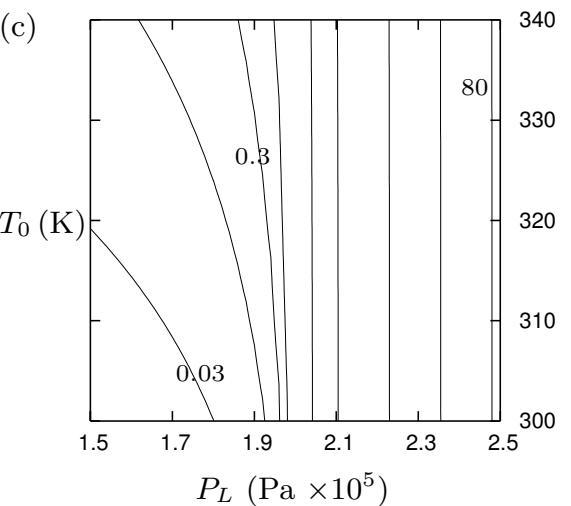

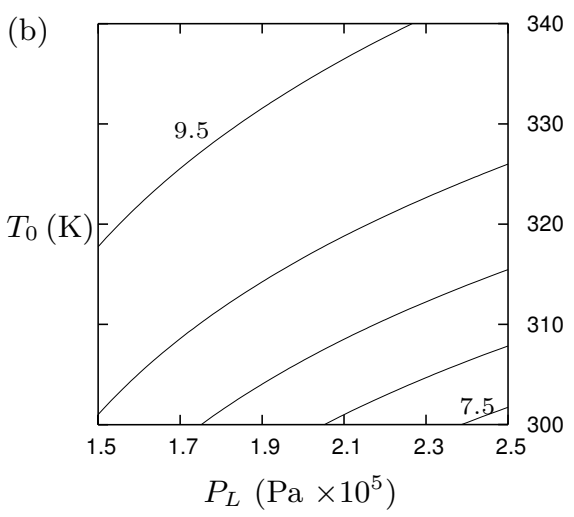

(d)

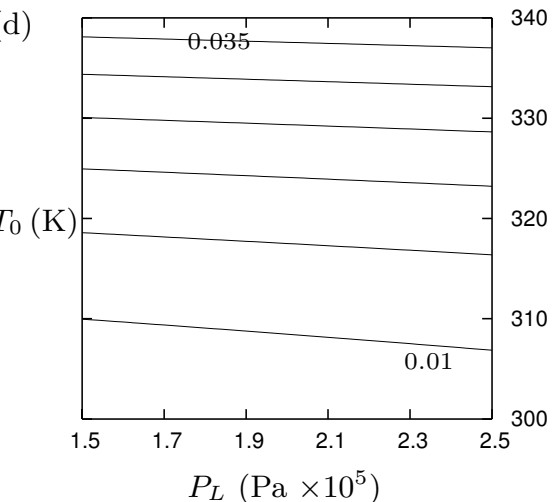

FIGURE 2. The steady-state solution for a high- and a low-permeability system: (a) evaporation surface depth $h(\mathrm{~m})$ for $k=10^{-13} \mathrm{~m}^{2}$ (contours at $h=0.001,0.003,0.01,0.1$ and 0.5 to 4.5 by $0.5)$; (b) evaporation surface depth $h(\mathrm{~m})$ for $k=10^{-18} \mathrm{~m}^{2}$ (contours at $h=7.5$ to 9.5 by 0.5 ); (c) upflow velocity $v_{0}\left(\mathrm{~mm} /\right.$ day) for $k=10^{-13} \mathrm{~m}^{2}$ (contours at $v_{0}=0.03,0.1,0.3,1,10$ and 20 to 80 by 20 ); (d) upflow velocity $v_{0}$ (mm/day) for $k=10^{-18} \mathrm{~m}^{2}$ (contours at $v_{0}=0.01$ to 0.035 by 0.005 ). In each case $L=10 \mathrm{~m}$ (so hydrostatic pressure corresponds to $P_{L}=1.98 \times 10^{5} \mathrm{~Pa}$ ), $\phi=0.3, \nu_{a}=0$, and other parameters have the standard values given in the text.

approximately linearly with $P_{L}$ and the evaporation surface is effectively located at the soil surface $h \approx 0$, so $v_{0} \approx k\left(P_{L}-P_{a}-\rho_{w} g L\right) /\left(\mu_{w} L\right)$ and $h / L \approx \beta / \lambda$. In these circumstances, the assumption of constant $\nu_{a}$ at the soil surface may be inappropriate as a very high evaporative flux will humidify the air immediately above the soil. When the aquifer is underpressured, the upflow velocity is very small and the evaporation surface position varies approximately linearly with $P_{L}, h / L \approx-\lambda$, so that the hydrostatic pressure at the base of the soil approximately matches the aquifer pressure $P_{L}$.

In the low-permeability system (figures $2 \mathrm{~b}$ and d), both $T_{0}$ and $P_{L}$ are significant; the influence of $T_{0}$ on $h$ is slightly stronger than that of $P_{L}$, and $T_{0}$ exerts the dominant effect on $v_{0}$. There is now no clear difference between the over- and underpressured regimes: as $P_{L}$ increases, this drives a greater upflow velocity and so $h$ decreases to provide a greater humidity gradient in the air-vapour region, and thus a greater vapour flux to the soil surface. As $T_{0}$ increases, $\nu_{*}$ and thus the evaporation rate increase: this drives higher upflow rates, but because the increase in $\nu_{*}$ strongly increases the humidity gradient, the 
evaporation surface in fact moves further from the soil surface, so $h$ increases with $T_{0}$ in this regime. We also note that the upflow velocities $v_{0}$ are of course much smaller in the low-permeability system, being of the order of $0.1 \mathrm{~mm}$ per day, compared to tens of centimetres per day in the high-permeability system.

As a final point, we recall that the results presented above were obtained assuming a dry atmosphere, $\nu_{a}=0$. The effect of changing the atmospheric humidity is similar to that of changing the system temperature, since all quantities calculated depend on $\nu_{*}\left(T_{0}\right)-\nu_{a}$. Consequently, in the high-permeability soil, we may expect a weak effect of $\nu_{a}$ on the front position and upflow, whereas in the low-permeability soil it becomes considerably more important: increasing $\nu_{a}$, like lowering $T_{0}$, will tend to bring the evaporation front closer to the soil surface and to decrease the upflow of water, while reducing $\nu_{a}$ will tend to move the evaporation front further from the surface and to increase upflow. In the extreme case $\nu_{a}=\nu_{*}$, there is of course no evaporation front within the soil, and any upflow is entirely pressure-driven.

We have presented this stationary solution in order to illustrate the evaporation process and to calculate the reduction of the water-table level and changes in the upflow water velocity. We will now consider the full problem when the upflowing groundwater is somewhat saline.

\section{Evaporation of saline groundwater}

In this section we derive the basic system when the mass of dissolved salt causes significant variations in the density of the saline groundwater. Our approach throughout, following, for example, van Duijn et al. (1998) and Tsypkin (2003b), will be to seek the simplest rather than the most comprehensive description of the phenomenon: this contrasts with a number of other studies of salt transport in groundwater, which have employed considerably more sophisticated models (e.g. Hassanizadeh and Leijnse 1988). In particular, we will assume linear constitutive and transport equations, ignoring deviations at high concentrations (Hassanizadeh and Leijnse 1995), and neglect capillary and osmotic pressures.

The assumption of a constant system temperature $T_{0}$ is also worth reiterating briefly. In our stability analysis we will consider variations in water density between fresh water and water with a saturation concentration of salt. The variations in water density due to salt content are therefore of the order of as much as $200-300 \mathrm{~kg} \mathrm{~m}^{-3}$ : this contrasts with the variation of density due to temperature over a range of 300 to $340 \mathrm{~K}$, which is around $15 \mathrm{~kg} \mathrm{~m}^{-3}$ (Wagner \& Pruss 2002). To leading order, then, it is justifiable to neglect variations in density due to temperature and to focus solely on the dominant effect of salt concentration.

\subsection{Basic equations}

As before, we assume that the transport of vapour in the region above the front is purely diffusive, and may be written in terms of the humidity $\nu$ as equation (2.1). Below the front, we assume that the velocity satisfies Darcy's law

$$
\mathbf{v}=-\frac{k}{\phi \mu_{s}}\left(\nabla P-\rho_{s} g \mathbf{e}_{z}\right),
$$

where $\mathbf{e}_{z}$ is the unit vector directed vertically downwards, and where $\rho_{s}$ and $\mu_{s}$ are, respectively, the density and dynamic viscosity of the saline groundwater. In the spirit of seeking the simplest physical model, we will assume that $\mu_{s}$ is constant, ignoring deviations at higher concentrations (Hassanizadeh and Leijnse 1988). 
To complete the set of governing equations we require equations relating the density and salinity of the groundwater and describing the transport of the water and salt components. Rigorously derived models for these processes can become very complex (e.g. Hassanizadeh \& Leijnse 1988, 1995); in the spirit of our approach we make some assumptions which lead to a relatively tractable model and which should capture the essential physics of the processes. We assume that the density $\rho_{s}$ is linearly related to the mass of dissolved salt $\rho_{c}$ per unit volume of the solution, so

$$
\rho_{s}=\rho_{0}+\alpha \rho_{c}=\rho_{c}+\rho_{w}
$$

where $\rho_{w}$ is the (variable) mass of water per unit volume, and where $\rho_{0}=1000 \mathrm{~kg} \mathrm{~m}^{-3}$ and $\alpha \approx 0.64$ (using the data presented by Herbert et al. 1988) are constants. This is equivalent to the 'additive rule' for solution density which Herbert et al. (1988) showed provides a good approximation to their tabulated data; although it may become inaccurate very close to the saturation density, our working assumption is that this should not distort the basic physics.

We further assume that the diffusive transport of $\rho_{s}$ and $\rho_{c}$ is Fickian (so we ignore deviations at higher concentrations: see e.g. Hassanizadeh and Leijnse 1995) and that the molecular diffusivities of the two components have the same constant value $D_{c}$. (Typically $D_{c} \approx 1.6 \times 10^{-9} \mathrm{~m}^{2} \mathrm{~s}^{-1}$; see e.g. Lide 2001.) We may then write

$$
\phi \frac{\partial \rho_{s}}{\partial t}+\phi \mathbf{v} \cdot \nabla \rho_{s}=\phi D_{c} \nabla^{2} \rho_{s}
$$

and we observe that by linearity $\rho_{c}$ and $\rho_{w}$ obey the same transport equation as $\rho_{s}$, while we maintain a divergence-free velocity field, $\nabla \cdot \mathbf{v}=0$.

\subsection{Boundary and initial conditions}

We recall that we assume that a narrow evaporation front migrates away from the ground and separates regions saturated with salty water and an air-vapour mixture (figure 1); the air-vapour region also contains precipitated salt in solid form, comprising part of the porous matrix. The boundary conditions at the front may be found using the conservation of mass of water and salt; we obtain

$$
\begin{aligned}
& {\left[1-\left(1-\frac{\phi_{p r}}{\phi}\right) \frac{\rho_{v *}}{\rho_{w *}}\right] \mathbf{V} \cdot \mathbf{n}=\mathbf{v} \cdot \mathbf{n}_{+}} \\
& \quad+D_{v} \frac{\rho_{a}}{\rho_{w *}}\left(1-\frac{\phi_{p r}}{\phi}\right)(\nabla \nu) \cdot \mathbf{n}_{-}-(1-\alpha) \frac{D_{c}}{\rho_{w *}}\left(\nabla \rho_{c}\right) \cdot \mathbf{n}_{+}
\end{aligned}
$$

and

$$
\left(1-\frac{\phi_{p r}}{\phi} \frac{\rho_{\text {salt }}}{\rho_{c *}}\right) \mathbf{V} \cdot \mathbf{n}=\mathbf{v} \cdot \mathbf{n}_{+}-\frac{D_{c}}{\rho_{c *}}\left(\nabla \rho_{c}\right) \cdot \mathbf{n}_{+} .
$$

Here an asterisk denotes a variable evaluated at the front; that is, since we assume instantaneous thermodynamic equilibrium at the front, a variable evaluated at its saturation value. The values for the saturation concentration $c_{*}=\rho_{c *} / \rho_{s *}$ tabulated by Lide (2001) may be approximated by

$$
c_{*}=0.34863-0.18494\left(\frac{T_{0}}{273.15}\right)+0.098963\left(\frac{T_{0}}{273.15}\right)^{2} .
$$

The solubility varies rather weakly over the relevant system temperature range: at $T_{0}=$ $300 \mathrm{~K}$ we find $c_{*} \approx 0.265$ and hence, using our linear equation of state, $\rho_{s *} \approx 1204 \mathrm{~kg} \mathrm{~m}^{-3}$, 
$\rho_{c *} \approx 319 \mathrm{~kg} \mathrm{~m}^{-3}$ and $\rho_{w *} \approx 885 \mathrm{~kg} \mathrm{~m}^{-3}$; while at $T_{0}=340 \mathrm{~K}$ we find $c_{*} \approx 0.272$ and hence $\rho_{s *} \approx 1211 \mathrm{~kg} \mathrm{~m}^{-3}, \rho_{c *} \approx 329 \mathrm{~kg} \mathrm{~m}^{-3}$ and $\rho_{w *} \approx 882 \mathrm{~kg} \mathrm{~m}^{-3}$. The quantity $\rho_{\text {salt }} \approx 2165 \mathrm{~kg} \mathrm{~m}^{-3}$ is the density of precipitated (solid) salt, and $\phi_{p r}$ is the fraction of the soil just above the front which is occupied by precipitated salt; in general $\phi_{p r}$ must be found as part of the solution.

From the salt diffusion equation we may estimate the typical timescale for the diffusing front to affect the aquifer, $t=L^{2} / D_{c}$. If $L \sim 1 \mathrm{~m}$ then $t \sim 10^{9} \mathrm{~s}$, or about 30 years. Thus, as $D_{c}$ is small and the diffusion process is very slow, it is natural to investigate the salt redistribution in the semi-infinite space $z>0$. The ground surface is located at $z=0$ with humidity $\nu=\nu_{a}$, while as $z \rightarrow \infty$ we have

$$
\rho_{s}=\rho_{s 0}, \quad \rho_{c}=\rho_{c}=\rho_{c 0}, \quad \mathbf{v}=-v_{0} \mathbf{e}_{z} \quad\left(\text { where } \quad v_{0}>0\right) .
$$

\section{Solution and stability analysis for a front propagating at constant velocity}

We will now consider a simplified problem which is amenable to analysis and which provides some insight into the essential dynamics of the system. We will seek solutions in which the front propagates downwards at a constant velocity $V$, with a constant upflow $v_{0}$. These conditions may be realised, for example, when the atmospheric humidity decreases with time in an appropriate manner; however, we will make the further assumption that the dynamics of the air-vapour region are unimportant except insofar as they prescribe $V$. When we carry out the stability analysis, we will further assume that the front remains unperturbed. This assumption is motivated by the fact that the front will be stabilised both by gravity and by evaporation, as vapour overlies liquid water; consequently, we may expect the fastest-growing perturbations to be those which involve little or no perturbation to the front. Our analysis resembles that by van Duijn et al. (2002), but is generalised to allow for a moving front.

It is important to note that the linear analysis which we present here does not give the last word on the stability of the system. As in many other convection problems (Straughan 1992), subcritical nonlinear instabilities may be possible below the threshold of linear instability, and this is indeed observed in the analysis of van Duijn et al. (2002). This phenomenon lies beyond the scope of the current study, but is an important direction for future treatments of the problem.

\subsection{Non-dimensional formulation}

We define the non-dimensional variables

$$
\begin{gathered}
S=\frac{\rho_{s}-\rho_{s 0}}{\rho_{s *}-\rho_{s 0}}=\frac{\rho_{c}-\rho_{c 0}}{\rho_{c *}-\rho_{c 0}}, \quad z=\frac{D_{c}}{v_{0}+V} \zeta, \quad x=\frac{D_{c}}{v_{0}+V} \hat{x}, \quad y=\frac{D_{c}}{v_{0}+V} \hat{y}, \\
t=\frac{D_{c}}{\left(v_{0}+V\right)^{2}} \tau, \quad \mathbf{v}=\frac{k g}{\phi \mu_{s}}\left(\rho_{s *}-\rho_{s 0}\right) \mathbf{u}, \quad P=\frac{D_{c}}{v_{0}+V}\left(\rho_{s *}-\rho_{s 0}\right) g \Pi+\rho_{s 0} g z .
\end{gathered}
$$

The non-dimensionalised governing equations are

$$
\begin{gathered}
\mathbf{u}=-\hat{\nabla} \Pi+S \mathbf{e}_{z}, \\
\frac{\partial S}{\partial \tau}+R_{s} \mathbf{u} \cdot \hat{\nabla} S=\hat{\nabla}^{2} S, \\
\hat{\nabla} \cdot \mathbf{u}=0,
\end{gathered}
$$

where a caret ^ denotes differentiation with respect to dimensionless variables, and where 
$R_{s}$ is the Rayleigh number

$$
R_{s}=\frac{k}{\phi \mu_{s}} \frac{\rho_{s *}-\rho_{s 0}}{v_{0}+V} g
$$

defined over the boundary-layer thickness of the salt concentration profile. We note that this is almost identical to the Rayleigh number defined by van Duijn et al. (2002), with the exception that the effective upflow velocity now becomes $V+v_{0}$.

It is then convenient to eliminate some components of the velocity $\mathbf{u}=(u, v, w)$ from the problem. Combining equations (4.2) and (4.4) we may write

$$
\hat{\nabla}^{2} \Pi=\frac{\partial S}{\partial \zeta} .
$$

Combining the $z$-component of equation (4.2) with equation (4.6) yields

$$
\hat{\nabla}^{2} w=\left(\frac{\partial^{2}}{\partial \hat{x}^{2}}+\frac{\partial^{2}}{\partial \hat{y}^{2}}\right) S
$$

It is then convenient to define a new vertical co-ordinate travelling with the front,

$$
\xi=\zeta-\frac{V}{v_{0}+V} \tau
$$

Defining $\nabla^{\prime}=\left(\partial_{\hat{x}}, \partial_{\hat{y}}, \partial_{\xi}\right)$, the set of governing equations now becomes

$$
\begin{gathered}
\nabla^{\prime 2} \Pi=\frac{\partial S}{\partial \xi} \\
\nabla^{\prime 2} w=\left(\frac{\partial^{2}}{\partial \hat{x}^{2}}+\frac{\partial^{2}}{\partial \hat{y}^{2}}\right) S \\
\frac{\partial S}{\partial \tau}-\frac{V}{v_{0}+V} \frac{\partial S}{\partial \xi}+R_{s}\left(-\frac{\partial \Pi}{\partial \hat{x}} \frac{\partial S}{\partial \hat{x}}+-\frac{\partial \Pi}{\partial \hat{y}} \frac{\partial S}{\partial \hat{y}}+w \frac{\partial S}{\partial \xi}\right)=\nabla^{\prime 2} S .
\end{gathered}
$$

The boundary conditions written in terms of non-dimensionalised variables are

$$
\begin{gathered}
S=1 \quad \text { at } \xi=0 ; \\
\left(1-\frac{\phi_{p r}}{\phi} \frac{\rho_{\text {salt }}}{\rho_{c *}}\right) V=R_{s}\left(v_{0}+V\right) w-\frac{\rho_{c *}-\rho_{c 0}}{\rho_{c *}}\left(v_{0}+V\right) \frac{\partial S}{\partial \xi} \quad \text { at } \quad \xi=0 ; \\
S \rightarrow 0 \quad \text { and } \quad w \rightarrow-w_{0}=-\frac{v_{0}}{R_{s}\left(v_{0}+V\right)} \quad \text { as } \quad \xi \rightarrow \infty .
\end{gathered}
$$

\subsection{Stationary solution}

We now seek a stationary solution below the front, in which $w=w_{s t}=-w_{0}$ is constant, $S=S_{s t}(\xi)$ and $\Pi=\Pi_{s t}(\xi)$.

Equation (4.11) now reduces to

$$
-\frac{\mathrm{d} S_{s t}}{\mathrm{~d} \xi}=\frac{\mathrm{d}^{2} S_{s t}}{\mathrm{~d} \xi^{2}}
$$

Applying the boundary conditions at $\xi=0$ and as $\xi \rightarrow \infty$, we find

$$
S_{s t}=\exp (-\xi)
$$

similarly, we may obtain $\Pi_{s t}=w_{0} \xi+\exp (-\xi)$ to within an additive constant (which for our purposes is irrelevant). 
Note that from the relation $\phi_{p r} / \phi<1$ and the boundary condition (3.5) we have

$$
V>\frac{\rho_{c 0}}{\rho_{\text {salt }}-\rho_{c 0}} v_{0} .
$$

This will be important in determining the appropriate physical parameter regime to explore.

\subsection{Linear stability analysis}

To analyse the linear stability of the stationary solution (4.16) we put

$$
S=S_{s t}+\delta S, \quad w=w_{0}+\delta w, \quad \Pi=\Pi_{s t}+\delta \Pi
$$

and linearise (4.9), (4.10) and (4.11) assuming that these perturbations are small, to obtain

$$
\begin{gathered}
\nabla^{\prime 2} \delta w=\left(\frac{\partial^{2}}{\partial \hat{x}^{2}}+\frac{\partial^{2}}{\partial \hat{y}^{2}}\right) \delta S, \\
\frac{\partial \delta S}{\partial \tau}+\frac{\partial \delta S}{\partial \xi}-R_{s} \exp (-\xi) \delta w=\nabla^{\prime 2} \delta S .
\end{gathered}
$$

The boundary conditions become

$$
\begin{gathered}
\bar{\rho} R_{s} \delta w=\frac{\partial \delta S}{\partial \xi} \quad \text { at } \quad \xi=0, \quad \text { where } \quad \bar{\rho}=\frac{\rho_{c *}}{\rho_{c *}-\rho_{c 0}}, \\
\delta S \rightarrow 0 \quad \text { and } \quad \delta w \rightarrow 0 \quad \text { as } \quad \xi \rightarrow \infty .
\end{gathered}
$$

The parameter $\bar{\rho}$ represents how large the salt content of the groundwater is just beneath the front compared to the difference in salt content between the front and the far field. The absolute (rather than relative) density is important in this problem because it enters into the conservation of salt at the front; this is the key difference between this problem and the classic stability analysis of a diffusive boundary layer with throughflow (Wooding 1960).

Setting

$$
\left.S=\hat{S}(\xi) \exp \left(\sigma \tau+i\left(a_{x} \hat{x}+a_{y} \hat{y}\right)\right), \quad w=w \hat{(\xi}\right) \exp \left(\sigma \tau+i\left(a_{x} \hat{x}+a_{y} \hat{y}\right)\right)
$$

with $a=\sqrt{a_{x}^{2}+a_{y}^{2}}$, we obtain the coupled equations

$$
\begin{gathered}
\left(\frac{\mathrm{d}^{2}}{\mathrm{~d} \xi^{2}}-a^{2}\right) \hat{w}=a^{2} \hat{S} \quad \text { and } \\
\left(\sigma-\frac{\mathrm{d}}{\mathrm{d} \xi}-\frac{\mathrm{d}^{2}}{\mathrm{~d} \xi^{2}}+a^{2}\right) \hat{S}-a^{2} R_{s} \exp (-\xi) \hat{w}=0 .
\end{gathered}
$$

We may then combine (4.22) and (4.23) to obtain the boundary value problem

$$
\left(\frac{\mathrm{d}^{2}}{\mathrm{~d} \xi^{2}}+\frac{\mathrm{d}}{\mathrm{d} \xi}-a^{2}-\sigma\right)\left(\frac{\mathrm{d}^{2}}{\mathrm{~d} \xi^{2}}-a^{2}\right) \hat{w}=a^{2} R_{s} \exp (-\xi) \hat{w}
$$

subject to the boundary conditions

$$
\begin{gathered}
\left(\frac{\mathrm{d}^{2}}{\mathrm{~d} \xi^{2}}-a^{2}\right) \hat{w}=0, \quad\left(\frac{\mathrm{d}^{3}}{\mathrm{~d} \xi^{3}}-a^{2} \frac{\mathrm{d}}{\mathrm{d} \xi}+a^{2} \bar{\rho} R_{s}\right) \hat{w}=0 \quad \text { at } \xi=0 \\
\text { and } \hat{w} \rightarrow 0 \quad \text { as } \quad \xi \rightarrow \infty .
\end{gathered}
$$

Let $R_{s}^{1}(a, \sigma)$ be the lowest positive eigenvalue of the generalized spectral problem 
defined by (4.24), (4.25) and (4.26). If $R_{s}>R_{a}^{1}(a, 0)$, then there exists some $\sigma>0$ such that $R_{s}=R_{s}^{1}(a, \sigma)$, i.e. there exists a growing infinitesimal perturbation. If $R_{s}^{1}(a, \sigma)<$ $R_{s}^{1}(a, 0)$ then $\sigma<0$ (see van Duijn et al. 2002). In other words, if, for a fixed value of $\bar{\rho}$, the Rayleigh number $R_{s}$ lies above the neutral curve $R_{s}=R_{s}^{1}(a, 0)$, then there exists a growing infinitesimal perturbation, implying that the front given by (4.16) is unstable. Meanwhile the domain below the neutral curve corresponds to decaying infinitesimal perturbations.

To determine the family of of neutral curves $R_{s}=R_{s}^{1}(a, \sigma)$ for different values of $\bar{\rho}$, we use the method of descending exponentials (Wooding 1960). We consider equation (4.24) for $\sigma=0$,

$$
\left(\frac{\mathrm{d}^{2}}{\mathrm{~d} \xi^{2}}+\frac{\mathrm{d}}{\mathrm{d} \xi}-a^{2}\right)\left(\frac{\mathrm{d}^{2}}{\mathrm{~d} \xi^{2}}-a^{2}\right) \hat{w}=a^{2} R_{s}(0) \exp (-\xi) \hat{w}
$$

and seek solutions in the form

$$
\hat{w}=\sum_{n=0}^{\infty} R_{s}^{n}\left(A A_{n} \exp [-(\beta+n) \xi]+B B_{n} \exp [-(\gamma+n) \xi]\right)
$$

where $A$ and $B$ are arbitrary constants, and $A_{n}, B_{n}, n=0,1,2, \ldots$ are constants to be determined from equation (4.27). We normalise the series by setting $A_{0}=B_{0}=1$, and we define $\beta=a$ and $\gamma=\frac{1}{2}+\sqrt{\frac{1}{4}+a^{2}}$, so the condition in the far field, equation (4.26), is satisfied. Substituting (4.28) into (4.27) indicates that the coefficients $A_{n}, B_{n}$ satisfy the recurrence relations

$$
\begin{aligned}
\frac{A_{n+1}}{A_{n}} & =\frac{a^{2}}{\left[(\beta+n+1)^{2}-(\beta+n+1)-a^{2}\right]\left[(\beta+n+1)^{2}-a^{2}\right]}, \\
\frac{B_{n+1}}{B_{n}} & =\frac{a^{2}}{\left[(\gamma+n+1)^{2}-(\gamma+n+1)-a^{2}\right]\left[(\gamma+n+1)^{2}-a^{2}\right]} .
\end{aligned}
$$

Substituting (4.28) into the boundary conditions at $\xi=0$, equations (4.25), we obtain the homogeneous system of linear equations

$$
\begin{gathered}
A \sum_{n=0}^{\infty} R_{s}^{n} A_{n}\left[(\beta+n)^{2}-a^{2}\right]+B \sum_{n=0}^{\infty} R_{s}^{n} B_{n}\left[(\gamma+n)^{2}-a^{2}\right]=0 \\
A \sum_{n=0}^{\infty} R_{s}^{n} A_{n}\left[a^{2}(\beta+n)-(\beta+n)^{3}+a^{2} \bar{\rho} R_{s}\right]+B \sum_{n=0}^{\infty} R_{s}^{n} B_{n}\left[a^{2}(\gamma+n)-(\gamma+n)^{3}+a^{2} \bar{\rho} R_{s}\right]=0 .
\end{gathered}
$$

The existence of a non-trivial solution of the system (4.31) implies that the marginal values $R_{s}(a, 0, \bar{\rho})$ of the Rayleigh number $R_{s}$ must satisfy

$$
\left|\begin{array}{ll}
\sum_{n=0}^{\infty} R_{s}^{n} A_{n}\left[(\beta+n)^{2}-a^{2}\right] & \sum_{n=0}^{\infty} R_{s}^{n} B_{n}\left[(\gamma+n)^{2}-a^{2}\right] \\
\sum_{n=0}^{\infty} R_{s}^{n} A_{n}\left[a^{2}(\beta+n)-(\beta+n)^{3}+a^{2} \bar{\rho} R_{s}\right] & \sum_{n=0}^{\infty} R_{s}^{n} B_{n}\left[a^{2}(\gamma+n)-(\gamma+n)^{3}+a^{2} \bar{\rho} R_{s}\right]
\end{array}\right|=0 .
$$

This equation was solved approximately by truncating the series for some large number of terms and locating the real roots of the resulting polynomial in $R_{s}$ numerically; the lowest positive root was then identified and plotted. The results presented here were calculated using Maple 10. At large $a$ and $R_{s}$ we require a large number of terms in the series for 


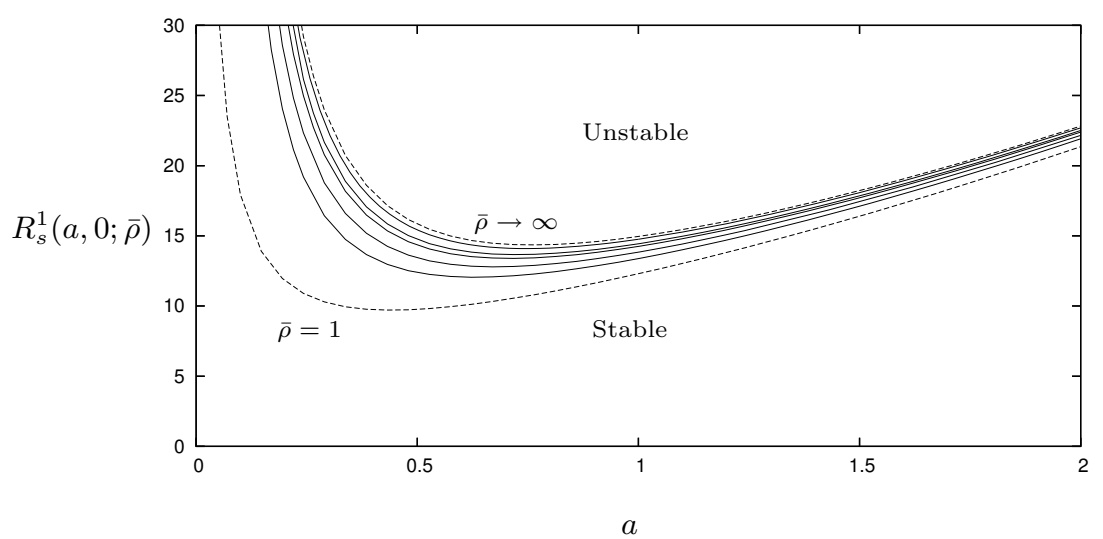

FiguRE 3 . The marginal stability curves $R_{s}^{1}(a, 0 ; \bar{\rho})$ as functions of wavenumber $a$, for $\bar{\rho}=1$ (lowest) $1.5,2,3,4,10$ and $\bar{\rho} \rightarrow \infty$ (highest). The dashed lines indicate the limiting cases $\bar{\rho}=1$ and $\bar{\rho} \rightarrow \infty$.

(a)

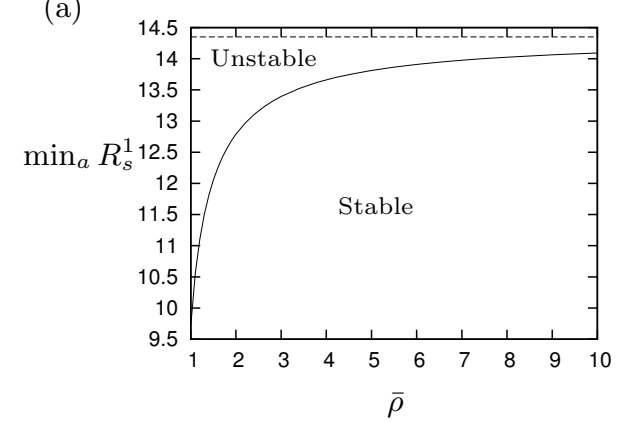

(b)

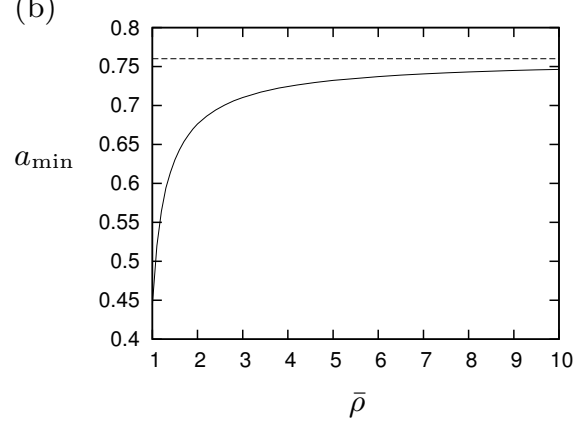

FiguRE 4. (a) The minimum over $a$ of the marginal Rayleigh number $R_{s}^{1}(a, 0 ; \bar{\rho})$ as a function of $\bar{\rho}$; the dashed line is the value $R_{s}^{1}=14.35$ for the limit $\bar{\rho} \rightarrow \infty$. (b) The corresponding values of $a$, representing the wavenumber of the most unstable perturbations; the dashed line is the value $a_{\min }=0.76$ for the limit $\bar{\rho} \rightarrow \infty$.

accuracy; however, the critical quantity which determines the stability of the system is the minimum over $a$ of the lowest branch of positive solutions $R_{s}^{1}(a, 0 ; \bar{\rho})$, and this always occurs at reasonably small values of $a$ and $R_{s}$. Numerical experimentation indicated that taking between 20 and 40 terms gave fully converged results in the parameter regimes which we consider here.

Figure 3 shows the marginal stability curves as functions of wavenumber. For any fixed value of $\bar{\rho}$, the marginal stability curve $R_{s}^{1}(a, 0)$ tends to infinity both for long waves $(a \rightarrow 0)$ and for short waves $(a \rightarrow \infty)$, and has a well-defined global minimum over $a$. This minimum occurs for values of $a$ of order unity, in other words for perturbations whose horizontal structure scales with the thickness of the saline boundary layer.

The variation of the stability condition with $\bar{\rho}$ (figure 4 a) is also easy to interpret. As $\bar{\rho}$ increases, the density contrast between the fluid just below the front and that at depth increases; with a greater density gradient driving the fingering instability, the system becomes unstable at lower values of the Rayleigh number. The most unstable wavenumber $a$ increases with $\bar{\rho}$ (figure $4 \mathrm{~b}$ ). The lowest value of the minimum Rayleigh 
number occurs when $\bar{\rho}=1$, and is given by $R_{s}^{1} \approx 9.71$; it corresponds to a wavenumber $a \approx 0.444$.

We note that in the formulation we have presented the limit $\bar{\rho} \rightarrow \infty$ is well-defined: this limit occurs as the salt content at depth, $\rho_{c 0}$, approaches its saturation value $\rho_{c *}$. In this limit, the salt conservation boundary condition becomes $\delta w=0$ at $\xi=0$ : this is formally identical to the classical stability problem for a diffusive boundary layer with throughflow (see e.g. van Duijn et al. 2002), and we may obtain a limiting curve $R_{s}(a)$ with its minimum at $a \approx 0.76, R_{s} \approx 14.35$ (the dashed curve in figure 3 ). We note, though, that in this limit $\left(\rho_{s *}-\rho_{s 0}\right) \rightarrow 0$, so although instability occurs at a finite Rayleigh number $R_{s}$ it requires very small values of $v_{0}+V$ to become unstable in this limit.

\subsection{Applications}

To illustrate the applicability, as well as the limitations, of our analysis, we now consider our stability predictions for a particular system similar to that considered in $\S 2$. As in that section, we consider a soil layer of finite depth, with upflow driven by a combination of evaporative draw-up and overpressure in the underlying aquifer. We use the expressions (2.13) and (2.14) derived there to estimate the upflow velocity $v_{0}$, neglecting any corrections due to the salinity of the water. The speed $V$ of the descending front is likely to vary in time, slowing as the front approaches its equilibrium depth (cf. the experiments of Rose, Konukcu \& Gowing 2005), and we recall that if $V$ becomes sufficiently small then the criterion (4.17) no longer holds, and the model breaks down because the soil just behind the front becomes completely clogged with salt. The question we will address is whether or not instability can occur before the front slows to this extent. To this end, we take the smallest permissible value of $V, V=v_{0} \rho_{c 0} /\left(\rho_{\text {salt }}-\rho_{c 0}\right)$ and thus the highest value which $R_{s}$ may attain. If this value of $R_{s}$ lies above the stability boundary $R_{s}^{1}(\bar{\rho})$ then instability may occur; if this value of $R_{s}$ lies below the stability boundary then we may be confident that instability does not occur before the soil has become clogged with salt. (We note that by considering this particular problem in a layer of finite depth we have excluded, for example, the possibility that the front might propagate downwards indefinitely leaving the soil above it only partially clogged with salt; however, this restriction allows us to specify the system in terms of directly observable physical conditions.)

As in $\S 2$, we will hold most of the parameters of the problem fixed, with the same values as used to plot figure 2. We will investigate the effect of altering the aquifer pressure $P_{L}$, the far-field solution density $\rho_{s 0}$, the humidity $\nu_{a}$ and the soil permeability $k$. Figure 5 shows the lines $R_{s}=R_{s}^{1}(\bar{\rho})$ plotted in the $\left(P_{L}, \rho_{s 0}\right)$ plane and in the $\left(P_{L}, \nu_{a}\right)$ plane for various values of $k$.

The most obvious feature of figure 5 is that our model never predicts instability when the aquifer is significantly overpressured $\left(P_{L} \gtrsim 2 \times 10^{5} \mathrm{~Pa}\right)$. We recall from figure $2 \mathrm{c}$ that when the aquifer is overpressured this drives a strong upflow $v_{0}$, which will tend to suppress instability. Another clear feature of these figures is slightly more surprising: as the permeability $k$ decreases, the region of possible instability shrinks, becoming confined to rather strongly underpressured aquifers and either very low values of $\rho_{s 0}$ or very high values of the relative humidity $\nu_{a} / \nu_{*}$, before disappearing altogether. This is despite the fact that the stabilising upflow velocity declines with decreasing $k$ (figures $2 \mathrm{c}$ and $\mathrm{d}$ ); this effect is overcome by the general decrease in the Rayleigh number, which we recall is proportional to $k$.

It is notable that the system becomes more unstable with increasing $\nu_{a}$ (figure $5 \mathrm{~b}$ ), as this reduces the stabilising upflow. This figure also indicates that when the other 

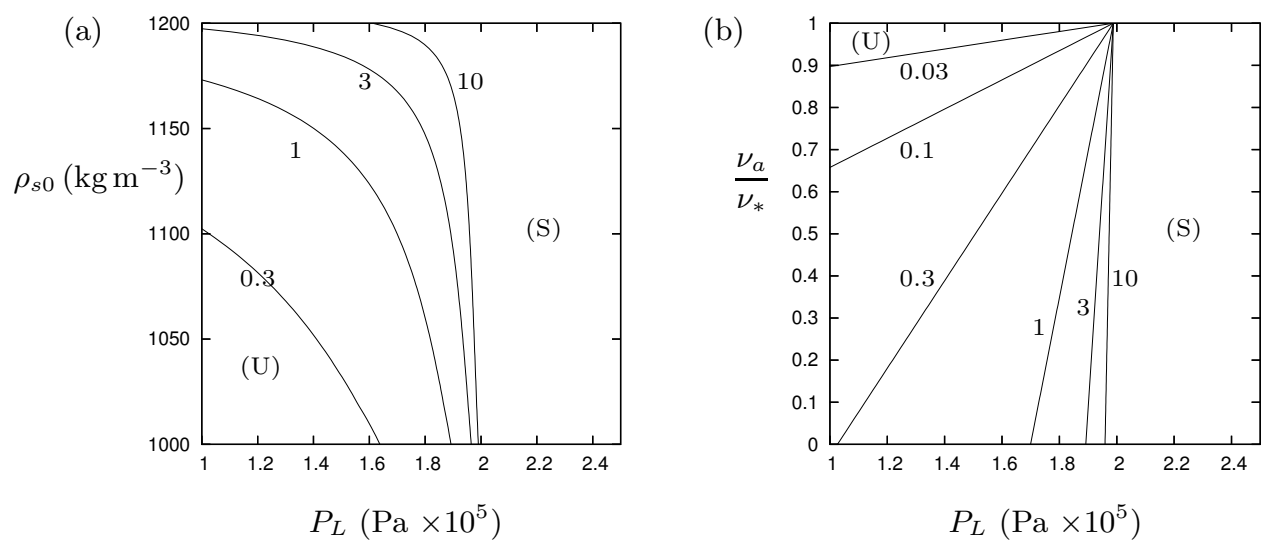

Figure 5. (a) Stability boundaries in the $\left(P_{L}, \rho_{s 0}\right)$ plane for $\nu_{a}=0$. (b) Stability boundaries in the $\left(P_{L}, \nu_{a}\right)$ plane for $\rho_{s 0}=1100 \mathrm{~kg} \mathrm{~m}^{-3}$. The labels $(\mathrm{S})$ and $(\mathrm{U})$ represent the regimes in which the system is, respectively, stable (so we may expect clogging with salt) and unstable (so we may expect fingering). The system temperature $T_{0}=340 \mathrm{~K}$ in each case; labels indicate permeability $k$ in $\mathrm{m}^{2} \times 10^{-14}$.

parameters are fixed, the marginal curve $\nu_{a}\left(P_{L}\right)$ is almost a straight line. This is because the marginal stability condition becomes $R_{s}=$ constant and hence $v_{0}=$ constant; now $v_{0} \propto\left(\nu_{*}-\nu_{a}\right) / h$ from equation (2.14), and in this regime $h$ is effectively pressurecontrolled, becoming a linear function of $P_{L}$ (cf. $\left.\S 2.2\right)$; hence the marginal stability condition becomes a linear relation between $\nu_{a}$ and $h$.

\section{Discussion and conclusions}

In this study we have used an approach based on conservation laws to develop some simple models which aim to capture aspects of the process of groundwater evaporation and upflow, and of the inception of salt fingering which may occur below a descending evaporation front. These models are motivated by environmental problems such as the intensification of groundwater evaporation in response to river water level changes and soil salinisation due to seawater invasion of an aquifer. In the latter case the evaporation process may lead to the development of saline seeps, which are a major cause of salinisation of littoral regions.

Mathematically, our simplified model of gravitational instability below a descending front is closely related to the classical problem of the stability of a diffusive boundary layer with upflow (Wooding 1960). The principal difference is that in our problem, the conservation of salt across the front introduces a mixed boundary condition, containing the extra parameter $\bar{\rho}$ which involves the absolute salinity of the groundwater. This mixed boundary condition tends to make the system slightly less stable, so the result of Wooding (1960) provides an upper limit for the Rayleigh number $R_{s}$ in our problem; nevertheless, our results are similar to those in the classical problem. In particular, we find an overall minimum for the Rayleigh number corresponding to marginal stability, $R_{s} \approx 9.71$ when $\bar{\rho}=1$, so regardless of the value of $\bar{\rho}$ we expect that infinitesimal perturbations will decay if $R_{s} \lesssim 9.71$; conversely we expect instability if $R_{s} \gtrsim 14.35$, regardless of the value of $\bar{\rho}$. (It is, however, possible that some perturbations may grow transiently even below the linear stability boundary, as found for the classical problem by Pieters \& van Duijn 2006, and also that subcritical nonlinear instabilities may occur.) 
An analysis of the steady, horizontally uniform solutions to the model for fresh water (§2) reveals two main regimes of behaviour. When the soil is highly permeable, the dominant influence is the aquifer pressure: when the aquifer is significantly underpressured (i.e. when $P_{L}$ is rather smaller than $\rho_{w} g L$ ) the front locates itself so that the hydrostatic pressure at the base of the soil approximately matches the aquifer pressure, and the upward flux of water is rather small; conversely, when the aquifer is overpressured $\left(P_{L} \gtrsim \rho_{w} g L\right)$ there is a large pressure-driven upflow and the front locates itself close to the soil surface. In this latter case our model is likely to break down as the air just above the soil becomes humidified by the large vapour flux, so the simple boundary condition $\nu=\nu_{a}$ cannot be maintained and a more complicated coupled problem must be considered. When the soil is less permeable, the location of the evaporation surface is determined largely by the humidity gradient in the upper part of the soil, and becomes sensitive to system temperature and to atmospheric humidity through the control they exert on the humidity gradient between the evaporation front and the soil surface.

Combining these results with the predictions of the linear stability analysis (§3), we conclude that the most significant effect controlling the stability is the permeability of the soil. If the permeability is very low (for example, a clay-rich soil with $k \approx 10^{-17}$ $\mathrm{m}^{2}$ ) then flow is very slow and incipient salt fingers cannot develop before being overcome by diffusion, so the system remains stable for all physically reasonable conditions. Conversely, if the permeability is rather high (for example, a sandy soil with $k>10^{-16}$ $\mathrm{m}^{2}$ ), then either stability or fingering is possible depending on the conditions at the soil surface and in the aquifer. The climatic conditions (humidity and system temperature) exert a strong influence by controlling the humidity contrast between the soil surface and the evaporation front, and thus influencing the upflow and the position of the front: upflow is highest under dry, hot conditions. Upflow is also increased by a higher pressure in the aquifer: indeed, when the aquifer is overpressured a pressure-driven upflow is able to stabilise the system quite effectively. With an significantly under-pressured aquifer, hot dry conditions at the soil surface and a relatively shallow evaporation front are required in order to drive a substantial upflow which stabilises the system; otherwise we predict that fingering is possible.

There are, of course, many possible extensions of the work presented here. In particular, while we believe that the simple stability model we have considered is likely to capture the essential controls on saline fingering beneath an evaporation front, it would be valuable to analyse the stability of less idealised flows. The similarity solution presented by Tsypkin (2003b) for a travelling evaporation-precipitation front under time-varying upflow would be a natural candidate for such an analysis. It would also be of use to consider both less idealised scenarios and the nonlinear development of fingers, though in these cases it is probable that numerical simulation rather than analytical methods would be required. Notwithstanding this, we believe that the results which we have obtained provide a useful guide to the likelihood of salt fingering in real-world situations.

We would like to thank three anonymous referees for their constructive and helpful comments. We are grateful for the financial support of the Royal Society of London under an International Joint Project grant (ref. 2005/R1). DP acknowledges the support of NERC and EPSRC under an EMS postdoctoral fellowship (ref. NE/B50188X/1) and of the University of Strathclyde through a Faculty of Science Starter Grant (VA5525C). We also acknowledge the hospitality of each other's institutions during several reciprocal visits in 2006 and 2007. 


\section{REFERENCES}

Gowing, J. W., Konukcu, F. \& Rose, D. A. 2006 Evaporative flux from a shallow watertable: the influence of a vapour-liquid phase transition. J. Hydrol. 321, 77-89.

Hassanizadeh, S. M. \& LeiJnse, T. 1988 On the modelling of brine transport in porous media. Wat. Resour. Res. 24(3), 321-330.

Hassanizadeh, S. M. \& Leijnse, A. 1995 A non-linear theory of high-concentration-gradient dispersion in porous media. Adv. Wat. Res. 18(4), 203-215.

Helmig, R. 1997 Multiphase Flow and Transport Processes in the Subsurface. Springer-Verlag, Berlin.

Herbert, A. W., Jackson, C. P. \& Lever, D. A. 1988 Coupled groundwater flow and solute transport with fluid density strongly dependent upon concentration. Wat. Resour. Res. 24(10), 1781-1795.

LIDE, D. R. 2001 CRC Handbook of Chemistry and Physics (82nd ed.) Boca Raton. New York, Washington.

Phillips, O. M. 1991 Flow and Reactions in Permeable Rocks. Cambridge University Press.

Pieters, G. J. M. \& van Duijn, C. J. 2006 Transient growth in linearly stable gravity-driven flow in porous media. Eur. J. Mech. B Fluids. 25, 83-94.

Rose, D. A., Konukcu, F. \& Gowing, J. W. 2005 Effect of watertable depth on evaporation and salt accumulation from saline groundwater. Aust. J. Soil Res. 43, 565-573.

Schofield, R. V. \& KiRKBy, M. J. 2003 Application of salinization indicators and initial development of potential global salinization scenario under climate change. Global Biogeochem. Cycles 17(3), 1078.

Straughan, B. 1992 The Energy Method, Stability, and Nonlinear Convection. Springer-Verlag.

Tsypkin, G. G. \& Brevdo L. 1999 A phenomenological model of the increase in solute concentration in ground water due to evaporation. Transport Porous Media 37, 129-151.

Tsypkin, G. G. 2003a Mathematical model of salt precipitation due to groundwater evaporation. Dokl. Physics. 48, 198-201.

TsYPKIN, G. G. 2003b Accumulation and precipitation of salts during groundwater evaporation and flow. Fluid Dynamics 38, 900-907. (Translated from Izvestiya Ross. Acad. Nauk, Mekh. Zhid. i Gaza 6, 84-93.)

van Duijn, C. J., Peletier, L. A. \& Schotting, R. J. 1998 Brine transport in porous media: self-similar solutions. Adv. Wat. Resour. 22(3), 285-297.

van Duijn, C. J., Wooding, R. A., Pieters, G. M. \& van der Ploeg, A. 2002 Stability criteria for the boundary layer formed by throughflow at a horizontal surface of a porous medium. In Environmental Mechanics: Water, Mass and Energy Transfer in the Biosphere, ed. P. A. C. Raats, D. Smiles \& A. W. Wanrick. Geophysical Monograph 129, American Geophysical Union.

Vukalovitch, M. P. 1955 Thermodynamic Properties of Water and Water Vapour. Mashgiz, Moscow.

Wagner, W. \& Pruss, A. 2002 The IAPWS formulation 1995 for the thermodynamic properties of ordinary water substance for general and scientific use. J. Phys. Chem. Ref. Data 31(2), 387-535.

Wooding, R. A. 1960 Rayleigh instability of a thermal boundary layer in flow through a porous medium. J. Fluid Mech. 9, 183-192.

Wooding, R. A., Tyler, S. W. \& White, I. 1997 Convection in groundwater below an evaporating salt lake: 1. Onset of instability. Wat. Resour. Res. 33(6), 1199-1217.

YAkirevich, A., Berliner, P. \& Sorek, S. 1997 A model for numerical simulating of evaporation from bare saline soil. Wat. Resour. Res. 33(5), 1021-1033.

YEO, A. 1999 Predicting the interaction between the effects of salinity and climate change on crop plants. Scientia Horticulturae 78, 159-174. 